\title{
Methanol Intoxication Causing Bilateral Putaminal Necrosis
}

\author{
Md Atiquzzaman ${ }^{1 *}$, Mohammad Omar Faruq ${ }^{2}$, Md Ashikuzzaman Sohan ${ }^{3}$
}

A 30-year-old lady without significant past medical illness was admitted to an ICU in Dhaka with the complaints of altered level of consciousness and blurring of vision for 1 day. She had no history of fever, headache, vomiting, convulsion, head trauma. History revealed that she had consumed unknown amounts of unbranded alcohol 2 days earlier.

Clinical examination revealed a Glasgow Coma Scale scoreof E1V1M4, bilaterally equally dilated $(6 \mathrm{~mm})$ pupils, normal deep tendon reflexes and bilateral flexor planter responses. Immediate arterial blood gas analysis showed severe metabolic acidosis $\left(\mathrm{pH}\right.$ 6.96, $\mathrm{pCO}_{2} 27 \mathrm{~mm} \mathrm{Hg}, \mathrm{HCO}_{3}^{-}$ $6.3 \mathrm{mEq} / \mathrm{L}$ ). A non-contrast CT scan of head done2 hours after admission which revealed bilateral hypodensities in the putamen (Figure 1). Based on these, with a presumed diagnosis of putamen necrosis secondary to methanol intoxication, bicarbonate and ethanol were administered. Immediately she received one session of haemodialysis following which her level of consciousness improved. Eventually anon-contrast MRI of brain done 4 days after methanol intoxication, which also revealed bilateral putaminal necrosis (Figure 2A-D).

6 days later, the patient was transferred out of ICU in stable condition. At the time of discharge, her sensorium recovered completely, but her vision was finger counting at $1 \mathrm{ft}$.

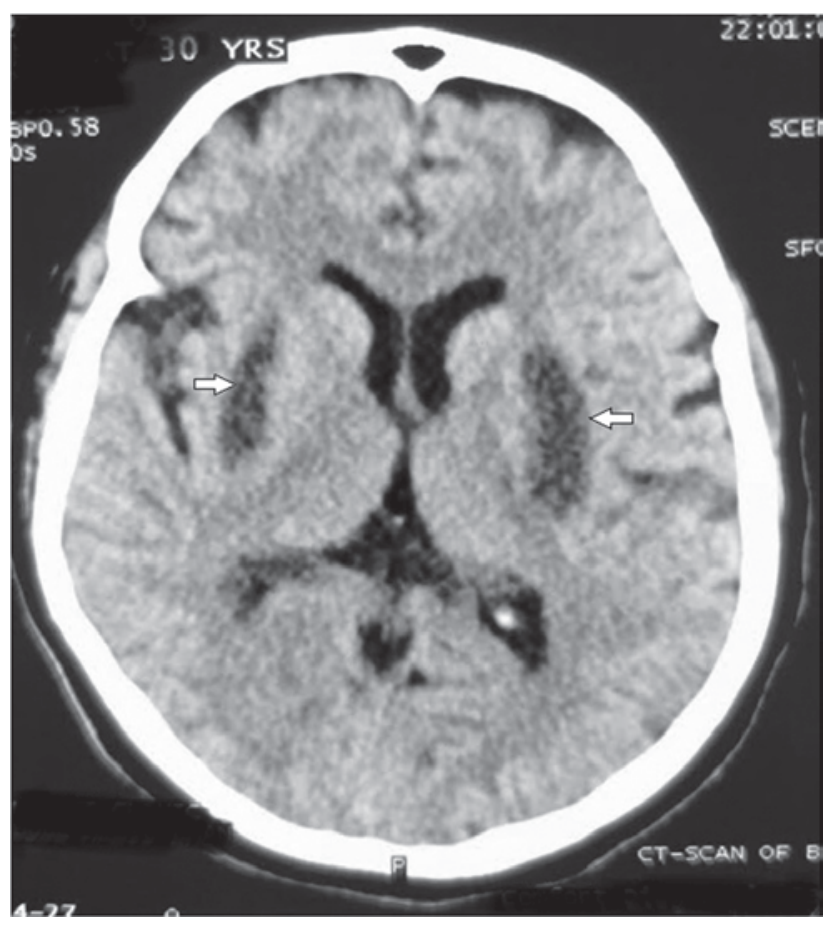

Figure 1: Non-contrast CT brain axial image showing bilateral putaminal hypodensity.

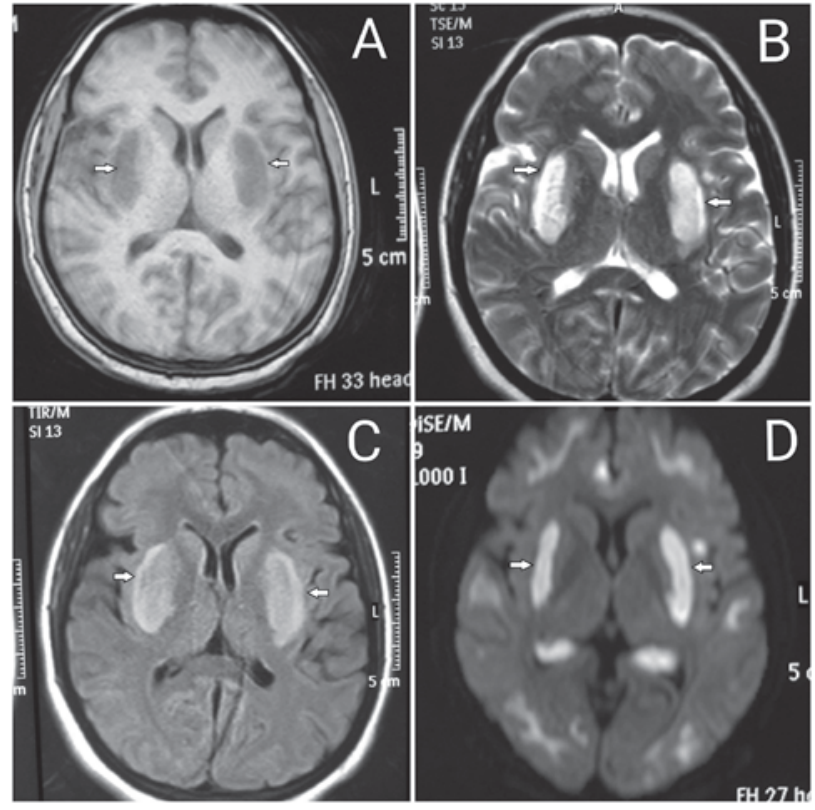

Figure 2: Brain MR Image without contrast showing bilateral symmetrical changes in the putamen. Hypointensity in T1W axialimage (2A), hyperintense signal changes in $\mathrm{T} 2 \mathrm{~W}$ axialimage (2B), FLAIR axial image (2C) \& diffusion restriction in DW axialimages (2D).

\section{Discussion:}

Methanol is a clear, colorless, highly toxic liquid with a smell and taste similar to alcohol. It is readily available as a component of many household products like antifreeze, paint removers, windshield washer fluid, various solvents and cleaners and gasoline mixtures ${ }^{1,3,5,6}$. It has also been used in fraudulent adulteration of alcoholic drinks ${ }^{6}$. Accidental or intentionalingestion leads to severe metabolic acidosis with associated serious neurological manifestations. Signs and symptoms of toxicity include nausea, vomiting, headache, dizziness and visual disturbances ranging from blurred vision to permanent blindness. As the metabolic acidosis worsens, respiratory failure and coma ensue ${ }^{1,5}$. Symptoms are usually observed 12-24 hour after methanol consumption. This latent period corresponds to the time period in which methanol is metabolized by alcohol dehydrogenase into formaldehyde and then to formic acid. It is the metabolite that lead to the toxic manifestations of methanol poisoning ${ }^{4,6}$.

History, neuro-ophthalmological symptoms, findings of metabolic acidosis with a high anion and osmolar gap are the diagnostic basis of methanol poisoning and high serum methanol levels further support the diagnosis 5 .

Neuroimaging is characteristic and diagnostic. Both CT and 
MRI give similar results but the latter yields better anatomical detail. Bilateral necrosis of the putamen with or without hemorrhage is the most characteristic radiological feature. Additional brain lesions including necrosis of the subcortical white matter, cerebellar cortical lesions, bilateral tegmental necrosis, diffuse cerebral edema, subarachnoid hemorrhage, cerebral hemorrhage and optic nerve necrosis have been described occasionally ${ }^{1,2,5,6}$.

Differential diagnosis of bilateral putamen necrosisincludes Wilson's disease, hypoxic-ischemic insults, encephalitis, Leigh disease, Kearns-Sayre syndrome, trichloroethane poisoning, striatal degeneration associated with Leber's optic atrophy, certain types of metabolic disorders, carbon monoxide inhalation and hypoxic-anoxic injuries such as near-drowning ${ }^{2,7}$. History of recent consumption of adulterated or non-standard alcohol is the main clue to proper diagnosis. Predilection to putamen not caudate helps to differentiate methanol poisoning from other conditions that tends to involve mainly the caudate nucleus ${ }^{7}$.

Treatment consists of inhibition of metabolism of methanol to toxic formic acid by competitive inhibition of the enzyme alcohol dehydrogenase by ethyl alcohol or 4-methyl pyrazole(fomepizole), alkalinization by administration of sodium bicarbonate, the use of cofactors such as folate and drug elimination (for example, haemodialysis) ${ }^{3,5}$.

1. Clinical Staff, General ICU, Asgar Ali Hospital, Dhaka-1204, Bangladesh.

2. Professor \& Chief Consultant, General ICU, United Hospital Ltd, Dhaka-1212, Bangladesh.

3. Senior Resident Medical Officer, General ICU, Asgar Ali Hospital, Dhaka-1204, Bangladesh.

*Corresponding Author:

Dr. Md. Atiquzzaman

MBBS

Clinical Staff, General ICU, Asgar Ali Hospital

111/1/A, Distillery Road, Gandaria, Dhaka-1204, Bangladesh.

E mail: drazrinku@gmail.com

Contact: +8801711939036

\section{References:}

1. Mark Glazer and Peter Dross. Necrosis of the Putamen Caused by Methanol Intoxication: MR Findings. American Journal of Roentgenology. 1993; 160: 1105-1106.

2. Ankur Gadodia, Bharti Singhal, Raju Sharma. Methanol intoxication causing putaminal necrosis. J Emerg Trauma Shock. 2011; 4(2): 300-301.

3. V Arora, IS Nijjar, H Thukral, Roopa. Bilateral Putaminal Necrosis Caused By Methanol Intoxication- A Case Report. Ind J RadiolImag. 2005; 15(3): 341-342.

4. Yashpal Singh, Gaurav Jain, and Dinesh K. Singh. Methyl alcohol poisoning causing putamen necrosis. Indian J Crit Care Med. 2014; 18(10): 698-699.

5. JSyuan-Huei Lin, Guo-Fang Tseng, Chung-Chao Liang, Yu-Chou Hung. Methanol Intoxication with Bilateral Putaminal and Occipital Necrosis. Tzu Chi Med J. 2010; 22(3): 160-163.

6. M. Blanco, R. Casado, F. Vázquez and J.M. Pumar. CT and MR Imaging Findings in Methanol Intoxication. American Journal of Neuroradiology. 2006; 27 (2): 452-454.

7. Sahar M. Elkhamary, Dalia M. Fahmy, Alberto Galvez-Ruiz, NasiraAsghar, Thomas M. Bosley. Spectrum of MRI findings in 58 patients with methanol intoxication: Long-term visual and neurological correlation. The Egyptian Journal of Radiology and Nuclear Medicine. 2016; 47(3): 1049-1055. 